\title{
Nonlinear Evolution of the Locally Induced Modulational Instability in Fiber Optics
}

\author{
Adrien E. Kraych, ${ }^{1}$ Pierre Suret, ${ }^{1}$ Gennady El, ${ }^{2}$ and Stéphane Randoux ${ }^{1, *}$ \\ ${ }^{1}$ University Lille, CNRS, UMR 8523-PhLAM-Physique des Lasers Atomes et Molécules, F-59000 Lille, France \\ ${ }^{2}$ Department of Mathematics, Physics and Electrical Engineering, Northumbria University, \\ Newcastle upon Tyne, NE1 8ST, United Kingdom
}

(Received 14 May 2018; published 6 February 2019)

\begin{abstract}
We report an optical fiber experiment in which we study the nonlinear stage of modulational instability of a plane wave in the presence of a localized perturbation. Using a recirculating fiber loop as the experimental platform, we show that the initial perturbation evolves into an expanding nonlinear oscillatory structure exhibiting some universal characteristics that agree with theoretical predictions based on integrability properties of the focusing nonlinear Schrödinger equation. Our experimental results demonstrate the persistence of the universal evolution scenario, even in the presence of small dissipation and noise in an experimental system that is not rigorously of an integrable nature.
\end{abstract}

DOI: 10.1103/PhysRevLett.122.054101

Modulational instability (MI), known as the BenjaminFeir instability in water waves, is a ubiquitous phenomenon in focusing nonlinear media that is manifested in the growth of small, long-wavelength perturbations of a constant background [1-9]. The linear stage of MI is characterized by an exponential growth of all the perturbations falling in the region of the Fourier spectrum below a certain cut-off wave number [5]. This simple picture ceases to be valid when the amplitude of the growing perturbation becomes comparable to the background, i.e., at the nonlinear stage of MI.

In the nonlinear regime, MI exhibits a rich spatiotemporal dynamics that has recently been the subject of significant interest in several areas of experimental and theoretical physics [10-20]. In this respect, the focusing one-dimensional nonlinear Schrödinger equation (1D NLSE) plays a prominent role as a universal mathematical model describing at leading order wave phenomena relevant to many fields of nonlinear physics such as, e.g., optics and hydrodynamics [21]. A particular scenario of the MI development strongly depends on the type of initial conditions considered. In the majority of the existing analytical, numerical, and experimental studies of MI, periodic or random initial modulations of a constant background have been considered [10-13,18-20,22]. For these types of initial conditions the nonlinear stage of the MI development was shown to be dominated by breatherlike structures such as the Akhmediev, Kuznetsov-Ma,

Published by the American Physical Society under the terms of the Creative Commons Attribution 4.0 International license. Further distribution of this work must maintain attribution to the author(s) and the published article's title, journal citation, and DOI.
Peregrine breathers and their generalizations. The role of NLSE breather solutions has been extensively discussed in recent years in the context of the formation of rogue waves $[23,24]$. A particular type of breather solutions of the 1D NLSE, the so-called superregular solitonic solutions, have been shown in Refs. $[25,26]$ to describe the development of a certain type of small localized perturbations of the plane wave [27]. In the inverse scattering theory of the 1D NLSE regular and superregular solitonic solutions have a discrete spectrum component associated with their soliton content $[25,26]$.

When a localized (and not necessarily small) initial perturbation of a plane wave has an arbitrary shape (within the class of "solitonless" perturbations with no discrete spectrum), it was recently shown using the inverse scattering transform solutions of the 1D NLSE that the nonlinear dynamics of MI is characterized by a longtime "hyperbolic" scenario, where a universal (not depending on the shape of the initial localized perturbation to leading order) nonlinear oscillatory structure develops and expands in time with finite speed [28-30]. In sharp contrast with the previously mentioned MI scenarios involving the formation of various breathers, this scenario involves the formation of a symmetric expanding nonlinear wave structure described by the modulated elliptic solution of the 1D NLSE. The modulation provides a gradual transition from a fundamental soliton (realized only asymptotically as a local approximation of the solution) resting at the center to smallamplitude dispersive waves propagating away from the center with linear group velocity. This universal modulated elliptic solution of the 1D NLSE saturating the MI was first obtained in Ref. [31] in the framework of the Whitham modulation theory [32].

It has been demonstrated in Ref. [33] that the qualitative behaviors found within the integrable NLSE framework are 
robust and the considered nonlinear stage of MI can also be found in a variety of other wave systems being not necessarily integrable. In view of the fundamental significance of the 1D NLSE and its generalizations, the question of the physical relevance of this universal scenario of the MI development and the possibility of its realization in an experiment are of major importance for nonlinear physics.

In this Letter, we report the experimental observations of the space-time dynamics of a modulationally unstable plane wave modified by two types of localized perturbations: a hump and a well. Using a recirculating fiber loop as the experimental platform, we observe the expansion of a nonlinear oscillatory structure going along with the amplification of the small optical noise that surrounds the perturbed plane wave at the initial stage. The behaviors observed experimentally are quantitatively very well described by the 1D NLSE with a small linear damping term, and our experimental observations reveal that the "hyperbolic" scenario considered in Refs. [28,29,31,33,34] exhibits robustness to noise, to weak dissipation, and to some deviations from integrability that are inherent in any experimental system.

Even though modern single mode fibers (SMFs) represent propagation media with very small linear losses (typically $\sim 0.2 \mathrm{~dB} / \mathrm{km}$ at the telecommunication wavelength of $1550 \mathrm{~nm}$ ), their attenuation cannot be considered as being fully negligible over propagation distances of a few kilometers. Many of the optical fiber experiments realized in recent years for the observation of breather solutions of the 1D NLSE have encompassed this constraint by using waves with an optical power of the order of $\sim 1 \mathrm{~W}$. With this power, the characteristic nonlinear length typically ranges between $100 \mathrm{~m}$ and $1 \mathrm{~km}$ so that singlepass propagation experiments reasonably well described by the 1D NLSE can be performed within propagation lengths between one to several kilometers. In all single-pass fiber geometries where Watt-level powers are required, the generated nonlinear structures have typical durations falling between $\sim 1$ and $\sim 10 \mathrm{ps}$. This requires the use of fast optical detection devices like optical sampling oscillocopes or time lenses [11,35-38]. Moreover, the observation of the space-time dynamics in single-pass optical fiber experiments often represents a difficult task since it relies on destructive cutback techniques [39] or, alternatively, on nonlinear digital holography methods $[40,41]$.

In our work, we have adopted another strategy by implementing a recirculating fiber loop that presents the significant advantage to provide real-time observation of the space-time dynamics of the optical wave. Recirculating fiber loops have been previously used under many circumstances in the context of optical fiber communication [42], in particular to demonstrate long-distance transmission of solitons [43-46]. Here, this fiber system is used to provide in a nondestructive way the real-time stroboscopic view of the "slow" evolution of the perturbed plane wave, round-trip after round-trip inside the fiber loop. In our recirculating fiber loop, the optical power is kept typically around only $\sim 10 \mathrm{~mW}$ and the propagation distances that are reached can be as large as hundreds of kilometers. With this experimental approach, all the physically relevant characteristic lengths and durations are rescaled by one or two orders of magnitude; i.e., the nonlinear length becomes of the order of $\sim 100 \mathrm{~km}$ and the typical duration of soliton structures becomes $\sim 50 \mathrm{ps}$. With such a timescale, the local perturbation of the plane wave can be relatively easily engineered by using standard fast electro-optic modulators (EOMs). Moreover, the detection part can be ensured by fast electronic devices like photodiodes and oscilloscopes.

Our experimental setup is schematically shown in Fig. 1. It consists of a recirculating fiber loop, i.e., a ring cavity made up of $\sim 4 \mathrm{~km}$ of SMF closed on itself by a 90/10 fiber coupler. The coupler is arranged in such a way that $90 \%$ of the intracavity power is recirculated. A wide light pulse of $\sim 100 \mathrm{~ns}$ having a square shape is perturbed by a small localized perturbation of $\sim 30 \mathrm{ps}$ and circulates in the counterclockwise direction inside the fiber loop. The perturbed square pulse is generated by modulating the power of a single-frequency laser operating at $1550 \mathrm{~nm}$ (see Supplemental Material [47]) and is periodically injected inside the loop with a period of $10 \mathrm{~ms}$, which is much larger than the cavity round-trip time of $\sim 20 \mu \mathrm{s}$. It is monitored by a fast photodiode (PD1) connected to an oscilloscope having an electrical bandwidth of $36 \mathrm{GHz}$.
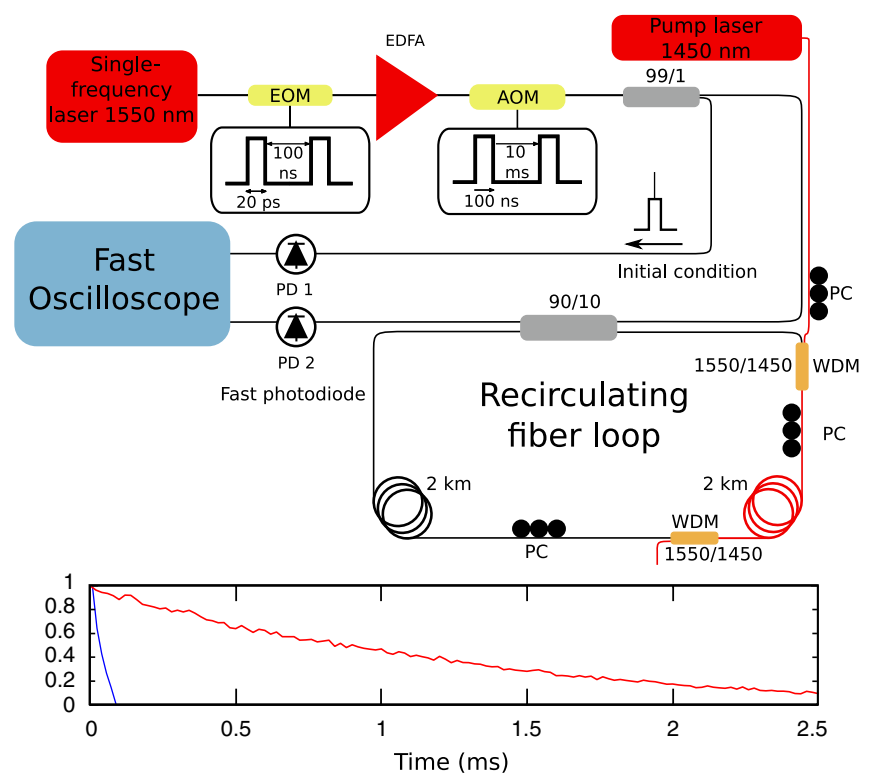

FIG. 1. Schematic representation of the experimental setup. PC, polarization controller; EDFA, erbium-doped fiber amplifier; AOM, acousto-optic modulator. The bottom part represents the decay of the plane wave power measured in the absence (blue line) and in the presence (red line) of Raman amplification at a pump power of $535 \mathrm{~mW}$. 
The linear losses of the SMF are around $\sim 0.2 \mathrm{~dB} / \mathrm{km}$. They are partially compensated by Raman amplification in a section of the loop that is $2 \mathrm{~km}$ long. Following the method used in Refs. [20,35], a pump beam at $1450 \mathrm{~nm}$ is launched in a counterpropagating (clockwise) direction to provide Raman gain with weak relative intensity noise. The pump power $P_{p}$ at $1450 \mathrm{~nm}$ is typically around $\sim 500 \mathrm{~mW}$, which is much greater than the power $\left(P_{0} \sim 10 \mathrm{~mW}\right)$ of the plane wave circulating inside the loop. The pump radiation at $1450 \mathrm{~nm}$ is coupled in and out of the fiber loop by using two wavelength dense multiplexers (WDMs). Increasing the power $P_{p}$ of the pump beam at $1450 \mathrm{~nm}$ from zero to a few hundreds of $\mathrm{mW}$, the decay time of the square pulse that propagates inside the loop and that is measured by the photodiode PD2 at the output of the fiber coupler dramatically increases from $\sim 40 \mu \mathrm{s}$ to $\sim 1 \mathrm{~ms}$, as shown in the bottom part of Fig. 1. Let us emphasize that our ring cavity is conceptually different and also simpler than coherently driven passive cavities used, e.g., in Refs. [49,50] as intrinsically bistable devices that can support dissipative cavity solitons.

Figure 2 (left) shows the space-time evolution of an optical plane wave initially perturbed by a localized bright (positive) perturbation having a duration of $\sim 30 \mathrm{ps}$ and a peak power twice as large as the mean power (14 $\mathrm{mW})$ of the plane wave; see Fig. 2(b). As shown in Fig. 2(a), the experiment reveals that a nonlinear oscillatory structure develops from the initial localized perturbation and expands with propagation distance, in qualitative agreement with the scenario theoretically described in
Refs. [28,29,31,33]. Additionally, the real-time single-shot measurement of the evolution of the perturbed plane wave reveals that the small optical noise that surrounds the plane wave at the initial stage [see Fig. 2(b)] is significantly amplified due to MI [see Figs. 2(a) and 2(c)].

In fact, Fig. 2 provides the experimental evidence of the inherent competition between the well-known process of noise amplification by MI and the process of the development of a nonlinear oscillatory structure within the wedgeshaped region. In our experiments we have observed that the nonlinear oscillatory structure shown Fig. 2(a) can be completely overtaken by the process of the exponential amplification of the small optical noise that perturbs the laser field at the initial stage. To obtain the space-time diagram of Fig. 2(a), the power $P_{0}$ of the initial plane wave and the power $P_{p}$ of the $1450 \mathrm{~nm}$ pump laser have been carefully adjusted to 14 and $535 \mathrm{~mW}$, respectively. With these values, the growth rate of the oscillatory structure that emerges from the local perturbation, the noise amplification rate, and the cavity loss rate are sufficiently well balanced for the nonlinear oscillatory structure to be observed over a propagation distance of $\sim 500 \mathrm{~km}$ [corresponding to $\sim 9$ times the nonlinear length $\left.L_{\mathrm{NL}}=1 /\left(\gamma P_{0}\right)\right]$. Taking some other values of $P_{0}$ and $P_{p}$, space-time evolutions similar to the one plotted in Fig. 2(a) can be observed over some larger or smaller physical propagation distances. However, the growth rate of the nonlinear oscillatory structure and the noise amplification rate are inherently comparable and it always happens that the nonlinear oscillatory structure never survives beyond a limited (typically between $\sim 5$
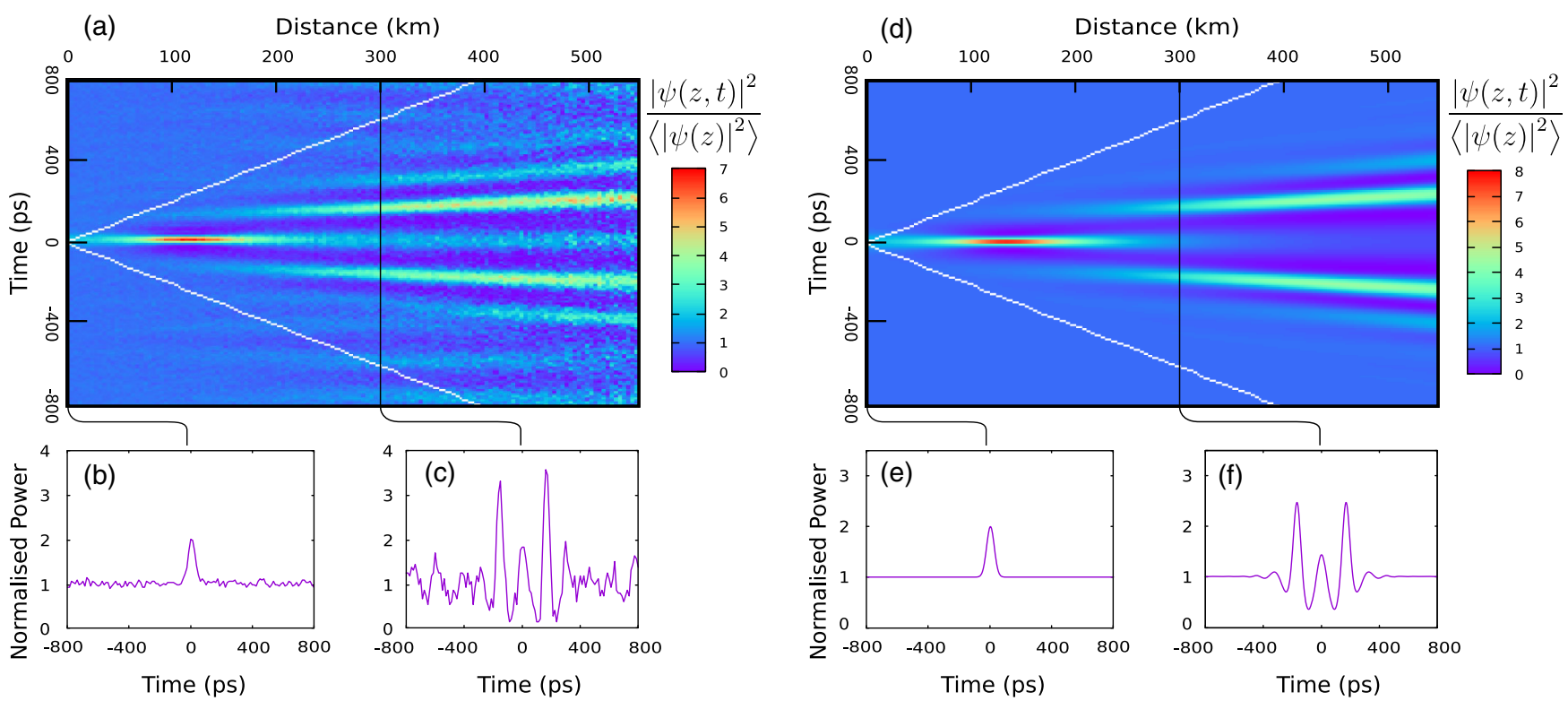

FIG. 2. Space-time evolution of a modulationally unstable plane wave perturbed at the initial stage by a localized bright (positive) peak. (a)-(c) Experiments and (d)-(f) numerical simulation of Eq. (1) with $\beta_{2}=-22 \mathrm{ps}^{2} \mathrm{~km}^{-1}, \gamma=1.3 \mathrm{~W}^{-1} \mathrm{~km}^{-1}, \alpha_{\mathrm{eff}}=$ $4.2 \times 10^{-3} \mathrm{~km}^{-1}, P_{0}=14 \mathrm{~mW}, T_{0}=30 \mathrm{ps}\left[\psi(z=0, t)=\sqrt{P_{0}\left[1+\exp \left(-\left(t / T_{0}\right)^{2}\right)\right]}\right]$. At each round-trip inside the cavity in (a) and (d), the optical power has been renormalized by the mean power carried by the exponentially decaying plane wave. $\left\langle|\psi(z)|^{2}\right\rangle=$ $P_{0} \exp \left(-\alpha_{\mathrm{eff}} z\right)$ represents the mean power of the plane wave at position $z$. 
and 10 ) number of nonlinear lengths before being destroyed by noise amplification. This feature has already been discussed and evidenced in simulations of the 1D NLSE where the numerical noise was shown to destroy the structure in finite time [29].

Behaviors found in the experiments are retrieved with a good quantitative agreement from the numerical integration of the 1D NLSE with small linear damping,

$$
i \frac{\partial \psi}{\partial z}=\frac{\beta_{2}}{2} \frac{\partial^{2} \psi}{\partial t^{2}}-\gamma|\psi|^{2} \psi-i \frac{\alpha_{\mathrm{eff}}}{2} \psi
$$

and with parameters corresponding to the experiments. $\psi(z, t)$ represents the complex envelope of the electric field that slowly varies in space $z$ and time $t$. At $1550 \mathrm{~nm}$ the group velocity dispersion coefficient of the SMF is $\beta_{2}=-22 \mathrm{ps}^{2} / \mathrm{km}$. The Kerr coefficient is $\gamma=$ $1.3 \mathrm{~W}^{-1} \mathrm{~km}^{-1}$ and the effective power losses $\alpha_{\text {eff }}$ measured from the decay rate of the plane wave inside the ring cavity are $4.2 \times 10^{-3} \mathrm{~km}^{-1}$ (equivalently $0.018 \mathrm{~dB} / \mathrm{km}$ ).

Figure 2 (right) shows the result of the numerical integration of Eq. (1) by taking $\psi(z=0, t)=$ $\sqrt{P_{0}\left[1+\exp \left(-\left(t / T_{0}\right)^{2}\right)\right]}$ as initial condition. Taking $P_{0}=14 \mathrm{~mW}$ and $T_{0}=30 \mathrm{ps}$, this expression fits quite well the experimental profile plotted in Fig. 2(b). There is a good quantitative agreement between right (numerical) and left (experimental) parts of Fig. 2, which indeed confirms that our experiment is well described by Eq. (1) where power losses have been introduced in a phenomenological way.

It has been shown in Refs. [28,29] that the development of the oscillation behavior in the nonlinear stage of MI does not depend on the exact shape of the localized perturbation,

(a)

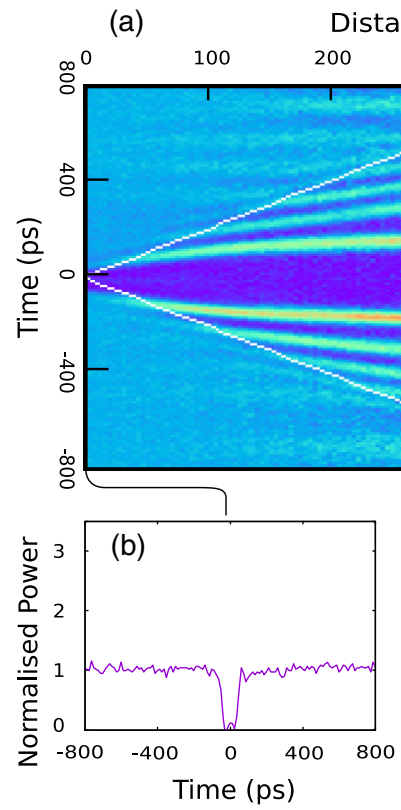

Distance $(\mathrm{km})$
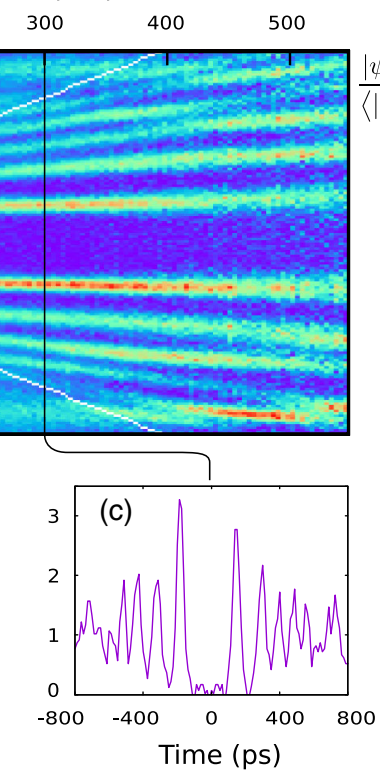

provided some (relatively mild) conditions necessary for a rigorous treatment are satisfied. We have investigated this point from our experiments. Figure 3 shows space-time evolution of an optical plane wave initially perturbed by a localized dark (negative) perturbation. The experimental results plotted in Figs. 3(a)-3(c) reveal that a nonlinear oscillatory structure grows from the initial perturbation. One can see that the detailed structure exhibiting two symmetric solitary waves separated by a narrow "vacuum" region in the central part slightly differs from the one observed in the positive perturbation case. However, the leading order modulation solution describing this structure is the same as in the positive perturbation case. This can be readily understood by noticing that the dynamics of the plane wave under sufficiently negative localized perturbation can be viewed as a combination of two focusing dam breaks of opposite signs located close to each other [15,51]. It follows from the results of Refs. [15,34,52] that the modulation solution describing such a "double dam break" problem is exactly the same as the one from Refs. [28,29,31] for the positive perturbation case [47], confirming the universality of the observed structure.

As shown in Fig. 3 (right), the behavior observed experimentally is also quantitatively well described by the numerical simulation of Eq. (1) taking $\psi(z=0, t)=$ $\sqrt{P_{0}\left[1-\beta \exp \left(-\left(t / T_{0}\right)^{4}\right)\right]}$ as the initial condition $\left(P_{0}=\right.$ $\left.16 \mathrm{~mW}, \beta=0.93, T_{0}=50 \mathrm{ps}\right)$ that fits the experimental profile shown in Fig. 3(b).

As shown in Refs. [28,31], the boundaries of the region separating the nonlinear oscillatory solution from the plane wave region are expanding linearly with the

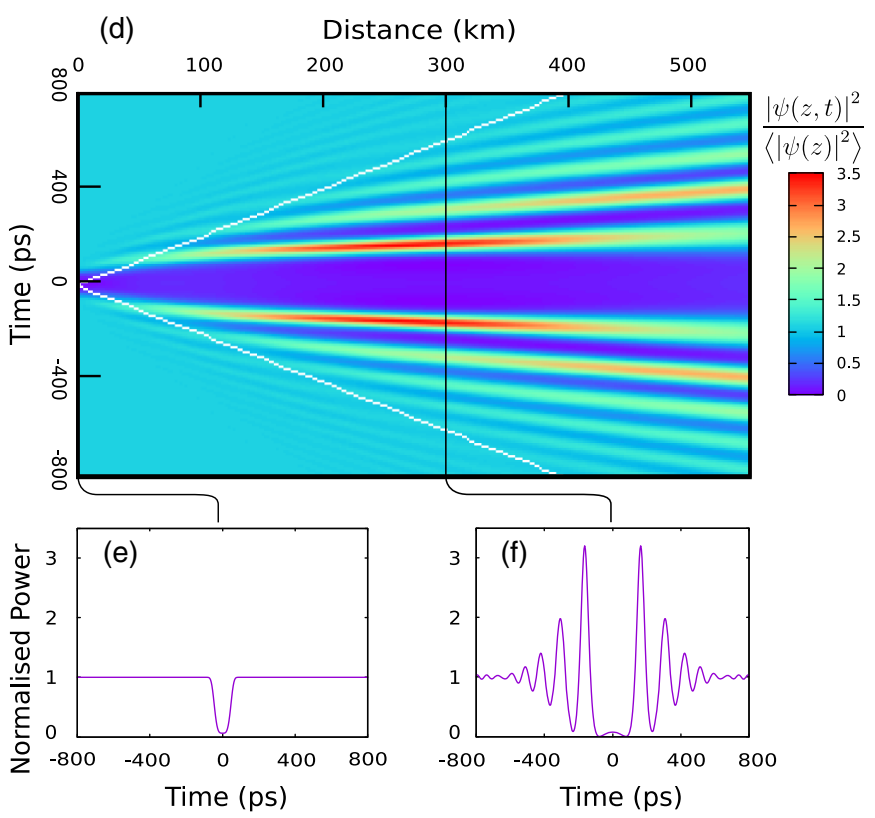

FIG. 3. Space-time evolution of a modulationally unstable plane wave perturbed at initial stage by a localized dark (negative) peak. (a)-(c) Experiments and (d)-(f) numerical simulation of Eq. (1) with $\beta_{2}=-22 \mathrm{ps}^{2} \mathrm{~km}^{-1}, \gamma=1.3 \mathrm{~W}^{-1} \mathrm{~km}^{-1}, \alpha_{\mathrm{eff}}=4 \times 10^{-3} \mathrm{~km}^{-1}$, $P_{0}=16 \mathrm{~mW}, T_{0}=50 \mathrm{ps}, \beta=0.93\left[\psi(z=0, t)=\sqrt{P_{0}\left[1-\beta \exp \left(-\left(t / T_{0}\right)^{4}\right)\right]}\right]$. 
evolution variable. When rephrased in physical variables, these boundaries are given by $t_{ \pm}= \pm 2 \sqrt{2 \beta_{2} \gamma P_{0}} z$ (see Supplemental Material [47]). They are plotted with white straight lines in Figs. 2(a), 2(d), 3(a), and 3(d). Even though the oscillatory structure is effectively located within the linear boundaries, the edges of the nonlinear oscillating structure are relatively far from the boundaries predicted by the asymptotic (longtime) theory. This quantitative difference between experiment and theory arises from the fact that the theoretical result has been established in the framework of the purely integrable and nondissipative 1D NLSE; see Supplemental Material [47]. Comparing Figs. 2(a) and 2(d) and Figs. 3(a) and 3(d), it is clear that dissipation has a stronger effect on the expansion of the bright perturbation than on the expansion of the dark one. At the qualitative level, this can be understood by realizing that the nonlinear wave structure establishes itself much faster due to the low background at the center, naturally fitting the rapidly decaying field in the fundamental soliton. Further theoretical work is needed to quantify the influence of small dissipation but also vectorial (polarization) effects on the universal oscillatory solution $[28,29,31,33]$ and its manifestation in experiment; see Ref. [53].

In summary, we have reported an optical fiber experiment in which we have observed the space-time dynamics of a modulationally unstable plane wave perturbed by a localized peak. Our experimental results demonstrate the robustness to noise and small dissipation of the expanding modulated solution theoretically found in Refs. [28,29,31]. Our experimental platform could be further used to explore other scenarios of the nonlinear stage of MI, including integrable turbulence or soliton gas [54-57].

This work has been partially supported by the Agence Nationale de la Recherche through the LABEX CEMPI project (ANR-11-LABX-0007), the Ministry of Higher Education and Research, Hauts de France council and European Regional Development Fund (ERDF) through the Nord-Pas de Calais Regional Research Council, and the European Regional Development Fund (ERDF) through the Contrat de Projets Etat-Région (CPER Photonics for Society P4S). The work of G. E. was partially supported by EPSRC Grant No. EP/R00515X/1 and Dstl Grant No. DSTLX1000116851. The authors are grateful to Draka-Prysmian for supplying the fiber and L. Bigot, R. Habert, E. Andresen, and IRCICA-TEKTRONIX European Optical and Wireless Innovation Laboratory for technical support on the electronic devices. The authors are also grateful to A. Mussot, C. Naveau, and P. Szriftgiser for providing temporary access to a specific optical filter.

*stephane.randoux@univ-lille1.fr

[1] T. B. Benjamin and J. E. Feir, J. Fluid Mech. 27, 417 (1967).

[2] T. B. Benjamin, Proc. R. Soc. A 299, 59 (1967).

[3] L. I. Zagryadskaya and L. A. Ostrovskii, Radiophys. Quantum Electron. 11, 548 (1968).
[4] L. A. Ostrovskii and L. V. Soustov, Radiophys. Quantum Electron. 15, 182 (1972).

[5] V. Zakharov and L. Ostrovsky, Physica (Amsterdam) 238D, 540 (2009).

[6] K. Tai, A. Hasegawa, and A. Tomita, Phys. Rev. Lett. 56, 135 (1986).

[7] M. Soljacic, M. Segev, T. Coskun, D. N. Christodoulides, and A. Vishwanath, Phys. Rev. Lett. 84, 467 (2000).

[8] D. R. Solli, G. Herink, B. Jalali, and C. Ropers, Nat. Photonics 6, 463 (2012).

[9] J. Meier, G. I. Stegeman, D. N. Christodoulides, Y. Silberberg, R. Morandotti, H. Yang, G. Salamo, M. Sorel, and J. S. Aitchison, Phys. Rev. Lett. 92, 163902 (2004).

[10] M. Erkintalo, K. Hammani, B. Kibler, C. Finot, N. Akhmediev, J. M. Dudley, and G. Genty, Phys. Rev. Lett. 107, 253901 (2011).

[11] B. Kibler, J. Fatome, C. Finot, G. Millot, F. Dias, G. Genty, N. Akhmediev, and J. M. Dudley, Nat. Phys. 6, 790 (2010).

[12] B. Frisquet, B. Kibler, and G. Millot, Phys. Rev. X 3, 041032 (2013).

[13] N. Akhmediev and A. Ankiewicz, Phys. Rev. E 83, 046603 (2011).

[14] G. Biondini and E. Fagerstrom, SIAM J. Appl. Math. 75, 136 (2015).

[15] G. A. El, E. G. Khamis, and A. Tovbis, Nonlinearity 29, 2798 (2016).

[16] S. Coulibaly, E. Louvergneaux, M. Taki, and L. Brevdo, Eur. Phys. J. D 69, 186 (2015).

[17] M. Taki, A. Mussot, A. Kudlinski, E. Louvergneaux, M. Kolobov, and M. Douay, Phys. Lett. A 374, 691 (2010).

[18] B. Frisquet, B. Kibler, J. Fatome, P. Morin, F. Baronio, M. Conforti, G. Millot, and S. Wabnitz, Phys. Rev. A 92, 053854 (2015).

[19] O. Kimmoun, H. Hsu, H. Branger, M. Li, Y. Chen, C. Kharif, M. Onorato, E. Kelleher, B. Kibler, N. Akhmediev et al., Sci. Rep. 6, 28516 (2016).

[20] A. Mussot, C. Naveau, M. Conforti, A. Kudlinski, F. Copie, P. Szriftgiser, and S. Trillo, Nat. Photonics 12, 303 (2018).

[21] J. M. Dudley, F. Dias, M. Erkintalo, and G. Genty, Nat. Photonics 8, 755 (2014).

[22] J. M. Soto-Crespo, N. Devine, and N. Akhmediev, Phys. Rev. Lett. 116, 103901 (2016).

[23] V. Shrira and V. Geogjaev, J. Eng. Math. 67, 11 (2010).

[24] M. Onorato, S. Residori, U. Bortolozzo, A. Montina, and F. Arecchi, Phys. Rep. 528, 47 (2013).

[25] V. E. Zakharov and A. A. Gelash, Phys. Rev. Lett. 111, 054101 (2013).

[26] A. A. Gelash and V.E. Zakharov, Nonlinearity 27, R1 (2014).

[27] B. Kibler, A. Chabchoub, A. Gelash, N. Akhmediev, and V. E. Zakharov, Phys. Rev. X 5, 041026 (2015).

[28] G. Biondini and D. Mantzavinos, Phys. Rev. Lett. 116, 043902 (2016).

[29] G. Biondini, S. Li, and D. Mantzavinos, Phys. Rev. E 94, 060201 (2016).

[30] G. Biondini and D. Mantzavinos, Commun. Pure Appl. Math. 70, 2300 (2017).

[31] G. El, A. Gurevich, V. Khodorovskii, and A. Krylov, Phys. Lett. A 177, 357 (1993). 
[32] G. B. Whitham, Linear and Nonlinear Waves (John Wiley \& Sons, New York, 2011).

[33] G. Biondini, S. Li, D. Mantzavinos, and S. Trillo, SIAM Rev. 60, 888 (2018).

[34] A. Kamchatnov, Phys. Rep. 286, 199 (1997).

[35] G. Xu, M. Conforti, A. Kudlinski, A. Mussot, and S. Trillo, Phys. Rev. Lett. 118, 254101 (2017).

[36] P. Walczak, S. Randoux, and P. Suret, Phys. Rev. Lett. 114, 143903 (2015).

[37] P. Suret, R. El Koussaifi, A. Tikan, C. Evain, S. Randoux, C. Szwaj, and S. Bielawski, Nat. Commun. 7, 13136 (2016).

[38] M. Närhi, B. Wetzel, C. Billet, S. Toenger, T. Sylvestre, J.-M. Merolla, R. Morandotti, F. Dias, G. Genty, and J. M. Dudley, Nat. Commun. 7, 13675 (2016).

[39] A. Tikan, C. Billet, G. El, A. Tovbis, M. Bertola, T. Sylvestre, F. Gustave, S. Randoux, G. Genty, P. Suret et al., Phys. Rev. Lett. 119, 033901 (2017).

[40] M. Tsang, D. Psaltis, and F. G. Omenetto, Opt. Lett. 28, 1873 (2003).

[41] A. Tikan, S. Bielawski, C. Szwaj, S. Randoux, and P. Suret, Nat. Photonics 12, 228 (2018).

[42] E. Desurvire, M. Digonnet, and H. J. Shaw, Opt. Lett. 10, 83 (1985).

[43] L. F. Mollenauer and K. Smith, Opt. Lett. 13, 675 (1988).

[44] M. Nakazawa, E. Yamada, H. Kubota, and K. Suzuki, Electron. Lett. 27, 1270 (1991).
[45] A. G. Okhrimchuk, G. Onishchukov, and F. Lederer, J. Lightwave Technol. 19, 837 (2001).

[46] E. A. Golovchenko, J. M. Jacob, A. N. Pilipetskii, C. R. Menyuk, and G. M. Carter, Opt. Lett. 22, 289 (1997).

[47] See Supplemental Material at http://link.aps.org/ supplemental/10.1103/PhysRevLett.122.054101, which includes Ref. [48], for a discussion about modulational instability of a locally perturbed plane wave and the focusing dam break problem.

[48] G. El, Chaos 15, 037103 (2005).

[49] F. Leo, S. Coen, P. Kockaert, S.-P. Gorza, P. Emplit, and M. Haelterman, Nat. Photonics 4, 471 (2010).

[50] F. Copie, M. Conforti, A. Kudlinski, A. Mussot, and S. Trillo, Phys. Rev. Lett. 116, 143901 (2016).

[51] F. Audo, B. Kibler, J. Fatome, and C. Finot, Opt. Lett. 43, 2864 (2018).

[52] R. Jenkins and K. D. McLaughlin, Commun. Pure Appl. Math. 67, 246 (2014).

[53] S. Randoux, P. Suret, A. Chabchoub, B. Kibler, and G. El, Phys. Rev. E 98, 022219 (2018).

[54] V. E. Zakharov, Stud. Appl. Math. 122, 219 (2009).

[55] S. Randoux, F. Gustave, P. Suret, and G. El, Phys. Rev. Lett. 118, 233901 (2017).

[56] G. A. El and A. M. Kamchatnov, Phys. Rev. Lett. 95, 204101 (2005).

[57] A. A. Gelash and D. S. Agafontsev, Phys. Rev. E 98, 042210 (2018). 